\title{
Accurate Boundary Conditions for Exterior Problems in Gas Dynamics
}

\author{
By Thomas Hagstrom* and S. I. Hariharan**
}

\begin{abstract}
The numerical solution of exterior problems is typically accomplished by introducing an artificial, far field boundary and solving the equations on a truncated domain. For hyperbolic systems, boundary conditions at this boundary are often derived by imposing a principle of no reflection. However, waves with spherical symmetry in gas dynamics satisfy equations where incoming and outgoing Riemann variables are coupled. This suggests that 'natural' reflections may be important. We propose a reflecting boundary condition based on an asymptotic solution of the far field equations. We obtain nonlinear energy estimates for the truncated problem and present numerical experiments to validate our theory.
\end{abstract}

1. Introduction. Interesting and important problems in gas dynamics are often posed in exterior domains. Examples include the explosion of gas bubbles in various media and flows external to aircraft. An approach to the numerical solution of such problems is to restrict the computational domain to a finite region through the introduction of an artificial boundary. For large time computations interactions between the solution and the artificial boundary can strongly influence the results. The focus of this paper is the development of an accurate treatment of these conditions.

A variety of authors have invoked a principle of no reflection. Notable among these are Engquist and Majda [2] who studied the general linear case and Hedstrom $[8]$ and Thompson [10] who considered nonlinear hyperbolic systems. However, as pointed out by Gustafsson and Kreiss [3], conditions satisfied by the exact solution may involve reflections. The current study involves spherical waves which exhibit coupling between incoming and outgoing Riemann variables. One expects this coupling to result in natural reflections which should be accounted for in an efficient numerical treatment. Indeed, Thompson [10] documents the disappointing performance of nonreflecting conditions in such cases.

An alternate approach is to incorporate the asymptotic behavior of the solution in the far field. Conditions for linear problems based on far field asymptotics have been sucessfully employed by Bayliss and Turkel [1] and Hariharan and Bayliss [7]. Our procedure is to develop approximate solutions to the appropriate weakly nonlinear initial-boundary value problem in the region exterior to the computational domain. A condition is thus obtained which includes appropriate reflections at

Received October 9, 1987.

1980 Mathematics Subject Classification (1985 Revision). Primary 76N15, 65M99.

*Research partially supported by NASA while the author was visiting ICOMP, NASA Lewis Research Center.

${ }^{* *}$ Research supported by National Science Foundation grant No. DMS-8604047. 
the computational boundary. Conditions involving incoming waves generated by inhomogeneities in the discarded region have also been proposed by Gustafsson [4], $[5]$.

The particular equations under consideration are the Euler equations for spherically symmetric, isentropic fluid flow:

$$
\begin{gathered}
\frac{\partial \rho}{\partial t}+\frac{\partial z}{\partial r}=-\frac{2 z}{r} \\
\frac{\partial z}{\partial t}+\frac{\partial}{\partial r}\left[\frac{z^{2}}{\rho}+f(\rho)\right]=-2 \frac{z^{2}}{\rho r} .
\end{gathered}
$$

Here, $\rho$ is the density, $z$ is the momentum and $f(\rho)$ is the pressure. Initial conditions are

$$
\rho(r, 0)=\rho_{0}(r) \quad \text { and } \quad z(r, 0)=z_{0}(r), \quad r \geq r_{0} .
$$

We also assume that the computational boundary is located at $r=L\left(L>r_{0}\right)$ and that proper conditions at $r_{0}$ are specified. Finally, we assume that

$$
\rho_{0}(r)=\rho_{\infty} \quad \text { and } \quad z_{0}(r)=0, \quad r \geq L .
$$

The plan of this paper is as follows: In Section 2 we follow the construction presented by Whitham [11] to obtain asymptotic solutions in the far field and derive the boundary conditions. Nonlinear energy estimates are established for the resulting finite domain problem in Section 3. Section 4 contains a discussion of the numerical treatment of the boundary conditions. In Section 5 numerical experiments are presented for an idealized weak explosion problem. Our technique is shown to yield the correct steady state for values of $L$ significantly smaller than those required by the nonreflecting conditions. In the final section we propose extensions of our conditions for the truly three-dimensional case.

2. Derivation of Asymptotic Boundary Conditions. We find it convenient to work with equations involving Riemann variables. They are

$$
\begin{aligned}
& R=\frac{z}{\rho}+G(\rho), \\
& S=\frac{z}{\rho}-G(\rho) .
\end{aligned}
$$

Here,

$$
G(\rho)=\int \frac{\sqrt{f^{\prime}(\rho)}}{\rho} d \rho .
$$

Then Eqs. (1.1) and (1.2) take the form

$$
\begin{gathered}
\frac{\partial R}{\partial t}+\left(\frac{z}{\rho}+\sqrt{f^{\prime}(\rho)}\right) \frac{\partial R}{\partial r}=-\frac{2 \sqrt{f^{\prime}(\rho)} z}{\rho r} \\
\frac{\partial S}{\partial t}+\left(\frac{z}{\rho}-\sqrt{f^{\prime}(\rho)}\right) \frac{\partial S}{\partial r}=\frac{2 \sqrt{f^{\prime}(\rho)} z}{\rho r}
\end{gathered}
$$

Here we assume that for $r \geq L$,

$$
\sqrt{f^{\prime}(\rho)}>\frac{z}{\rho}
$$


That is, the flow is subsonic in the far field. Then $R$ and $S$ are, respectively, the outgoing and incoming Riemann variables. Note that the lower-order terms couple the equations for the Riemann variables. This means that an outgoing wave generates an incoming wave.

To derive the boundary conditions, we consider the initial-boundary value problem (2.4), (2.5) and (1.4) on the exterior domain $r \geq L$ with boundary condition

$$
R(L, t)=g(t)
$$

Solving this problem yields

$$
S(L, t)=\mathscr{F}[g(\cdot)] .
$$

That is the incoming variable is a functional of the outgoing variable.

Equations (2.6) and (2.7) represent an exact boundary condition at $r=L$. However, the explicit form of the functional $\mathscr{F}$ is not known in general. Therefore, we construct an asymptotic solution of the exterior problem valid for $L$ sufficiently large. (Similar constructions for steady state problems can be found in Hagstrom and Keller [6].) Consistent with the known far field behavior of solutions of the linearized equations, we expand $R$ and $S$ as follows:

$$
\begin{aligned}
& R(r, t)=R_{0}+\frac{R_{1}(r, t)}{r}+\frac{R_{2}(r, t)}{r^{2}}+\cdots, \\
& S(r, t)=S_{0}+\frac{S_{1}(r, t)}{r}+\frac{S_{2}(r, t)}{r^{2}}+\cdots .
\end{aligned}
$$

We note that

$$
R_{0}=-S_{0}=G\left(\rho_{\infty}\right)
$$

We further assume

$$
g(t)=R_{0}+\frac{H(t)}{L}
$$

for some function $H(t)$.

Equations for $R_{1}$ and $S_{1}$ are given by

$$
\begin{aligned}
& \frac{\partial R_{1}}{\partial t}+\left(\frac{R_{1}+S_{1}}{2 r}+\sqrt{f^{\prime}\left(\rho_{\infty}\right)}+P\left(\rho_{\infty}\right) \frac{\left(R_{1}-S_{1}\right)}{r}\right) \frac{\partial R_{1}}{\partial r}=0 \\
& \frac{\partial S_{1}}{\partial t}+\left(\frac{R_{1}+S_{1}}{2 r}-\sqrt{f^{\prime}\left(\rho_{\infty}\right)}-P\left(\rho_{\infty}\right) \frac{\left(R_{1}-S_{1}\right)}{r}\right) \frac{\partial S_{1}}{\partial r}=0 .
\end{aligned}
$$

Here,

$$
P(u)=\frac{u f^{\prime \prime}(u)}{4 f^{\prime}(u)} .
$$

Following Whitham [11] we have retained $\frac{1}{r}$ corrections to the characteristic speed to suppress nonuniformities in the expansion as $r$ approaches infinity. Note that the source terms are absent at this order, so $R_{1}$ and $S_{1}$ are Riemann invariants of the approximate equations (2.12) and (2.13). Since the characteristics corresponding to $S_{1}$ all originate at $t=0$, we deduce

$$
S_{1}(r, t)=0 .
$$


That is, we have a simple wave. Now Eq. (2.12) can be solved for $R_{1}$ using the method of characteristics. The differential equations for the characteristics are given by

$$
\frac{d t}{d r}=\left[\sqrt{f^{\prime}\left(\rho_{\infty}\right)}+\left\{\frac{1}{2}+P\left(\rho_{\infty}\right)\right\} \frac{R_{1}}{r}\right]^{-1} .
$$

Using the fact that $R_{1}$ is constant along the characteristics, we find that

$$
\begin{gathered}
R_{1}(r, t(r ; \tau))=H(\tau) \\
t(r ; \tau)=\tau+\frac{r-L-B(\tau) \ln \left[\frac{r+B(\tau)}{L+B(\tau)}\right]}{\sqrt{f^{\prime}\left(\rho_{\infty}\right)}}
\end{gathered}
$$

where we have introduced

$$
B(\tau)=\frac{H(\tau)\left[\frac{1}{2}+P\left(\rho_{\infty}\right)\right]}{\sqrt{f^{\prime}\left(\rho_{\infty}\right)}} .
$$

We remark that this solution may break down where characteristics intersect, in which case a shock must be fitted in. In order to compute the first nonvanishing correction to the incoming variable, we consider the equation for $S_{2}$,

$$
\frac{\partial S_{2}}{\partial t}-\left(\sqrt{f^{\prime}\left(\rho_{\infty}\right)}+\frac{\left(P-\frac{1}{2}\right) R_{1}}{r}\right) \frac{\partial S_{2}}{\partial r}=\sqrt{f^{\prime}\left(\rho_{\infty}\right)} R_{1}
$$

To solve Eq. (2.18) we make a change of variables in which $S_{2}$ is expressed as a function of $\tau$ and $r$. This yields the above equation in the form

$$
\left(\frac{1}{\sqrt{f^{\prime}\left(\rho_{\infty}\right)}} \frac{\partial \tau}{\partial t}-D(\tau, r) \frac{\partial \tau}{\partial r}\right) \frac{\partial S_{2}}{\partial \tau}-D(\tau, r) \frac{\partial S_{2}}{\partial r}=R_{1}(\tau)
$$

which we write as

$$
\frac{\partial S_{2}}{\partial \tau}-N(\tau, r) D(\tau, r) \frac{\partial S_{2}}{\partial r}=N(\tau, r) R_{1}(\tau)
$$

Here,

$$
N(\tau, r)=\sqrt{f^{\prime}\left(\rho_{\infty}\right)} \frac{\left[1-B^{\prime}(\tau)\left\{\ln \left[\frac{r+B(\tau)}{L+B(\tau)}\right]+\frac{(r-L) B(\tau)}{(L+B(\tau))(r+B(\tau))}\right\}\right]}{\left(1+\left[1-\frac{B(\tau)}{r+B(\tau)}\right] \frac{D(\tau, r)}{\sqrt{f^{\prime}\left(\rho_{\infty}\right)}}\right)}
$$

and

$$
D(\tau, r)=1+\frac{\left(P-\frac{1}{2}\right) H(\tau)}{\sqrt{f^{\prime}\left(\rho_{\infty}\right)} r} .
$$

Again we solve Eq. (2.20) by the method of characteristics. We integrate from the first characteristic, $\tau=0$, where $S_{2}=0$. We will only need to know $S_{2}$ at the boundary $r=L$. Thus we obtain

$$
S_{2}(L, \tau)=\int_{0}^{\tau} R_{1}(s) N(s, \xi(s ; \tau, L)) d s
$$

where

$$
\frac{d \xi}{d s}=-N D, \quad \xi(\tau ; \tau, L)=L
$$


To put Eq. (2.21) in a more convenient form, we differentiate with respect to $\tau$ :

$$
\frac{\partial S_{2}(L, \tau)}{\partial \tau}=R_{1}(\tau) N(\tau, L)+\int_{0}^{\tau} R_{1}(s) \frac{\partial N(s, \xi(s ; \tau, L))}{\partial \xi} \frac{\partial \xi}{\partial \tau} d s
$$

Now the integral term is of order $\frac{1}{r}$ due to the presence of $\frac{\partial N}{\partial \xi}$. Therefore we neglect it along with $\frac{1}{L}$ contributions to $N$. Using $\frac{\partial t}{\partial \tau}(L, \tau)=1, S=S_{0}+\frac{1}{r^{2}} S_{2}$ and $R_{1}(\tau)=L\left(R(L, t)-R_{0}\right)$, we finally obtain

$$
\left.\frac{\partial S}{\partial t}\right|_{r=L}=\frac{\sqrt{f^{\prime}\left(\rho_{\infty}\right)}\left(R(L, t)-R_{0}\right)}{2 L} .
$$

Equation (2.24) is the boundary condition we propose. We also note that the relationship

$$
\frac{z}{\rho}-G(\rho)=-G\left(\rho_{\infty}\right)+O\left(\frac{1}{r^{2}}\right)
$$

can be used to derive a number of asymptotically equivalent conditions. For example,

$$
\frac{\partial S}{\partial t}=\frac{z \sqrt{f^{\prime}\left(\rho_{\infty}\right)}}{L \rho}
$$

and

$$
\frac{\partial S}{\partial t}=\frac{\left(G(\rho)-G\left(\rho_{\infty}\right)\right) \sqrt{f^{\prime}\left(\rho_{\infty}\right)}}{L} .
$$

3. Energy Estimates. We now study the problem on the truncated region $\left[r_{0}, L\right]$, rewriting the field equations (1.1) and (1.2) in a convenient form. Moreover, we take $r_{0}=0$ for definiteness. Thus the problem under consideration is

$$
\begin{gathered}
\frac{\partial \rho}{\partial t}+\frac{1}{r^{2}} \frac{\partial}{\partial r}\left(r^{2} z\right)=0 \\
\frac{\partial z}{\partial t}+\frac{1}{r^{2}} \frac{\partial}{\partial r}\left(r^{2} \frac{z^{2}}{\rho}\right)+\frac{\partial}{\partial r}[f(\rho)]=0 .
\end{gathered}
$$

Initial conditions are

$$
\rho(r, 0)=\rho_{0}(r), \quad z(r, 0)=z_{0}(r), \quad r \geq 0 .
$$

Our boundary condition at $r=L$ has the integrated form

$$
\frac{z}{\rho}=G(\rho)-G\left(\rho_{\infty}\right)+\alpha \int_{0}^{t} \frac{z}{\rho} d s+\beta \int_{0}^{t}\left(G(\rho)-G\left(\rho_{\infty}\right)\right) d s .
$$

Corresponding, respectively, to $(2.24),(2.26)$ and (2.27) we have

$$
\begin{gathered}
\alpha=\beta=\frac{\sqrt{f^{\prime}\left(\rho_{\infty}\right)}}{2 L}, \\
\alpha=\frac{\sqrt{f^{\prime}\left(\rho_{\infty}\right)}}{L}, \quad \beta=0, \\
\alpha=0, \quad \beta=\frac{\sqrt{f^{\prime}\left(\rho_{\infty}\right)}}{L} .
\end{gathered}
$$


In addition, we need to introduce a finiteness condition at $r=0$, owing to the singularity of the equations (3.1) and (3.2). This is accomplished by demanding

$$
z(r, t) \rightarrow 0 \quad \text { as } r \rightarrow 0 .
$$

It is difficult to establish the well-posedness of initial-boundary value problems for nonlinear hyperbolic systems. We content ourselves with the derivation of bounds on the growth of the total energy of the system. The (physical) energy density is defined by

$$
E=\frac{1}{2} \frac{z^{2}}{\rho}+\rho e(\rho),
$$

where the internal energy $e$ satisfies

$$
e^{\prime}(\rho)=\frac{f(\rho)}{\rho^{2}}
$$

We also define

$$
\mathbf{q}=\nabla E
$$

Here the gradient is with respect to the variables $\rho$ and $z$. Then

$$
\mathbf{q}=\left(-\frac{1}{2} \frac{z^{2}}{\rho^{2}}+\frac{f^{\prime}(\rho)}{\gamma-1}, \frac{z}{\rho}\right)^{T}
$$

Taking the inner product of (3.1) and (3.2) with $\mathbf{q}$, we obtain

$$
\frac{\partial E}{\partial t}+\frac{1}{r^{2}} \frac{\partial}{\partial r} \Psi=0
$$

where

$$
\Psi=\left[r^{2} z\left(e(\rho)+\frac{f(\rho)}{\rho}\right)\right]+r^{2} \frac{z^{3}}{2 \rho^{2}}
$$

Now integrating $(3.13)$ over $[0, L]$ with the weight $r^{2}$ we obtain

$$
\Phi^{\prime}(t)+\left.\Psi\right|_{0} ^{L}=0
$$

where

$$
\Phi(t)=\int_{0}^{L} r^{2} E d r
$$

which is the total energy of the system. It is useful to rewrite $\Psi$ :

$$
\Psi=r^{2} \frac{z}{\rho}\left[\rho e+f+\frac{z^{2}}{2 \rho}\right] .
$$

Clearly, (3.8) implies that $\Psi=0$ ar $r=0$, leaving us with

$$
\Phi^{\prime}(t)=-\Psi(L, z(L, t), \rho(L, t)) .
$$

We now state the main theorem of this section. 
THEOREM 1. There exists a bounded function $\Gamma(t)$, depending only on $f$ and $\rho_{\infty}$, so that the total energy of any generalized solution of (3.1)-(3.4), (3.8) satisfies

$$
\Phi(t) \leq \Phi(0)+\Gamma(t)
$$

Proof. Using Fubini's theorem, it may easily be verified that (3.4) solved for $\frac{z}{\rho}$ yields

$$
\frac{z(L, t)}{\rho(L, t)}=G(\rho(L, t))-G\left(\rho_{\infty}\right)+(\alpha+\beta) \int_{0}^{t} e^{\alpha(t-s)}\left(G(\rho(L, s))-G\left(\rho_{\infty}\right)\right) d s
$$

Since the bracketed quantity in (3.16) is positive, the right-hand side of (3.17) can be positive only if $\frac{z}{\rho}$ is negative. As $\rho$ is nonnegative and $G$ is a nondecreasing function, a positive contribution to the total energy implies

$$
G(\rho) \leq G\left(\rho_{\infty}\right)(1+\Delta(t)), \quad \rho \leq G^{-1}\left(G\left(\rho_{\infty}\right)(1+\Delta(t))\right)
$$

and

$$
\left|\frac{z}{\rho}\right| \leq G\left(\rho_{\infty}\right)(1+\Delta(t))
$$

where

$$
\Delta(t)= \begin{cases}\frac{(\alpha+\beta)}{\alpha}\left(e^{\alpha t}-1\right), & \alpha \neq 0 \\ \beta t, & \alpha=0 .\end{cases}
$$

Substituting these inequalities into (3.17) and integrating from 0 to $t$ immediately yields the desired result. If we specialize to the case of a polytropic gas, $f(\rho)=k \rho^{\gamma}$, we may further conclude that $\Gamma$ grows algebraically if $(2.27)$ is employed, and exponentially in the other cases.

4. Numerical Procedure. In this section we briefly discuss a particular numerical implementation of the boundary conditions we have developed. We note that many different, stable implementations are possible. Those we present here are used in the numerical experiments which follow.

Introducing a uniform mesh,

$$
r_{i}=(i-1) \cdot \Delta r, \quad i=1, \ldots, N+1,
$$

we denote our approximate solution vector by

$$
U_{i}^{t}=\left(\begin{array}{l}
\rho\left(r_{i}, t\right) \\
z\left(r_{i}, t\right)
\end{array}\right)
$$

and also introduce notation for the approximate fluxes and sources:

$$
\begin{gathered}
F(U)=\left(\begin{array}{c}
z \\
\frac{z^{2}}{\rho}+f(\rho)
\end{array}\right), \\
H(U, r)=\left(\begin{array}{c}
\frac{2 z}{r} \\
\frac{2 z^{2}}{\rho r}
\end{array}\right) .
\end{gathered}
$$

We employ the two-step Lax-Wendroff method (Sod [9]) in the interior:

$$
\begin{aligned}
U_{i+1 / 2}^{t+\Delta t / 2}= & \frac{1}{2}\left(U_{i}^{t}+U_{i+1}^{t}\right)-\frac{\Delta t}{2 \Delta r}\left(F\left(U_{i+1}^{t}\right)-F\left(U_{i}^{t}\right)\right) \\
& -\frac{\Delta t}{2} H\left(\frac{1}{2}\left(U_{i}^{t}+U_{i+1}^{t}\right), r_{i+1 / 2}\right), \quad i=1, \ldots, N
\end{aligned}
$$




$$
\begin{aligned}
U_{i}^{t+\Delta t}= & U_{i}^{t}-\frac{\Delta t}{\Delta r}\left(F\left(U_{i+1 / 2}^{t+\Delta t / 2}\right)-F\left(U_{i-1 / 2}^{t+\Delta t / 2}\right)\right) \\
& -\Delta t H\left(\frac{1}{2}\left(U_{i+1 / 2}^{t+\Delta t / 2}+U_{i-1 / 2}^{t+\Delta t / 2}\right), r_{i}\right), \quad i=2, \ldots, N .
\end{aligned}
$$

This is second-order in space and time for smooth solutions. The boundary conditions are used to update the solution at the boundaries. At $r=L$, our conditions $(2.24),(2.26),(2.27)$ are all of the form

$$
\frac{\partial S}{\partial t}=Q(\rho, z)
$$

A second-order discretization of this is given by

$$
S\left(U_{N+1}^{t+\Delta t}\right)=S\left(U_{N+1}^{t}\right)+S\left(U_{N}^{t}\right)-S\left(U_{N}^{t+\Delta t}\right)+2 \Delta t Q\left(U_{N+1 / 2}^{t+\Delta t / 2}\right) .
$$

Note that all quantities on the right are available from the interior scheme. Another numerical condition is needed. We obtain it from the equation for the outgoing characteristic, (2.4). Writing it in the form

$$
\frac{\partial R}{\partial t}+C(\rho, z) \frac{\partial R}{\partial r}=W(\rho, z)
$$

we have the second-order discretization

$$
\begin{aligned}
(1+\bar{C}) R\left(U_{N+1}^{t+\Delta t}\right)= & R\left(U_{N+1}^{t}\right)+R\left(U_{N}^{t}\right)-R\left(U_{N}^{t+\Delta t}\right)+\bar{W} \\
& -\bar{C}\left(R\left(U_{N+1}^{t}\right)-R\left(U_{N}^{t}\right)-R\left(U_{N}^{t+\Delta t}\right)\right)
\end{aligned}
$$

where

$$
\bar{C}=\frac{C\left(U_{N+1 / 2}^{t+\Delta t / 2}\right) \Delta t}{\Delta r}
$$

and

$$
\bar{W}=2 \Delta t W\left(U_{N+1 / 2}^{t+\Delta t / 2}\right) .
$$

These equations yield updates of the Riemann variables at the artificial boundary. Equations (2.1) and (2.2) are inverted to update the primitive variables:

$$
\begin{gathered}
\rho\left(r_{N+1}, t+\Delta t\right)=G^{-1}\left(\frac{R-S}{2}\right), \\
z\left(r_{N+1}, t+\Delta t\right)=\frac{1}{2} \rho\left(r_{N+1}, t+\Delta t\right)(R+S) .
\end{gathered}
$$

At the origin, continuity of the velocity field requires

$$
z(0, t+\Delta t)=0 \text {. }
$$

Again, a numerical boundary condition is required which we obtain from the characteristic equation, (2.5). Writing it in the form

$$
\frac{\partial S}{\partial t}+\hat{C}(\rho, z) \frac{\partial S}{\partial r}=\hat{W}(\rho, z)
$$

we use the second-order approximation

$$
\begin{aligned}
(1-\tilde{C}) S\left(U_{1}^{t+\Delta t}\right)= & S\left(U_{1}^{t}\right)+S\left(U_{2}^{t}\right)-S\left(U_{2}^{t+\Delta t}\right)+\tilde{W} \\
& -\tilde{C}\left(S\left(U_{2}^{t}\right)-S\left(U_{1}^{t}\right)+S\left(U_{2}^{t+\Delta t}\right)\right)
\end{aligned}
$$


where

$$
\tilde{C}=\frac{\hat{C}\left(U_{3 / 2}^{t+\Delta t / 2}\right) \Delta t}{\Delta r}
$$

and

$$
\tilde{W}=2 \Delta t \hat{W}\left(U_{3 / 2}^{t+\Delta t / 2}\right)
$$

Given $S$, we can compute $\rho$ from

$$
\rho(0, t+\Delta t)=G^{-1}(-S) .
$$

This completes the update of $U_{i}^{t+\Delta t}$.

5. Numerical Experiments. Here we present some numerical calculations to validate the effectiveness of our boundary conditions. For purposes of comparison we include the condition resulting from Thompson [10]. We here list and label the different conditions that we have used for the computation. All of them have the form

$$
\frac{\partial}{\partial t}\left(\frac{z}{\rho}-G(\rho)\right)=Q
$$

where

$$
\begin{array}{ll}
Q=\frac{z \sqrt{f^{\prime}\left(\rho_{\infty}\right)}}{\rho L} & \text { (from Eq. (2.26)), } \\
Q=\frac{\sqrt{f^{\prime}\left(\rho_{\infty}\right)}}{L}\left(G(\rho)-G\left(\rho_{\infty}\right)\right) & \text { (from Eq. (2.27)), } \\
Q=\frac{\sqrt{f^{\prime}\left(\rho_{\infty}\right)}}{2 L}\left(R(L, t)-G\left(\rho_{\infty}\right)\right) & \text { (from Eq. (2.24)), } \\
Q=\frac{2 z \sqrt{f^{\prime}\left(\rho_{\infty}\right)}}{L} & \text { (from Thompson [10]). }
\end{array}
$$

A simple idealized explosion problem is considered in which the density is initialized to

$$
\rho_{0}(r)= \begin{cases}3, & r<1, \\ 1, & r>1,\end{cases}
$$

and the momentum is initialized to

$$
z_{0}(r)=0, \quad 0 \leq r \leq L .
$$

The true solution includes the propagation of a weak, decaying shock with the solution on the truncated region eventually approaching the steady state $\rho=1$ and $z=0$. We note that only this steady state is compatible with boundary conditions $\mathrm{HH} 2$ and $\mathrm{HH} 3$. By contrast, $\mathrm{HH} 1$ and Th are compatible with steady states at any density. In the graphs presented here the momentum and density are plotted against $r$ at different time steps. We use a spatial mesh width of $\Delta r=.05$ and a time step chosen so that the CFL number corresponding to sound speed of the compressed gas, $\sqrt{f^{\prime}(3)}$, is .25 . In the first case considered here the far field boundary is located at $5(L=5)$. Figures $1-4$ (a) show, at time steps 400,600 , 800 and 1000 , respectively, the results obtained from the computations using the condition HH1. Similarly for conditions $\mathrm{HH} 2, \mathrm{HH} 3$ and $\mathrm{Th}$ the results are reported in Figures 1-4 (b)-(d). As can be seen in the figures, the solutions are initially 

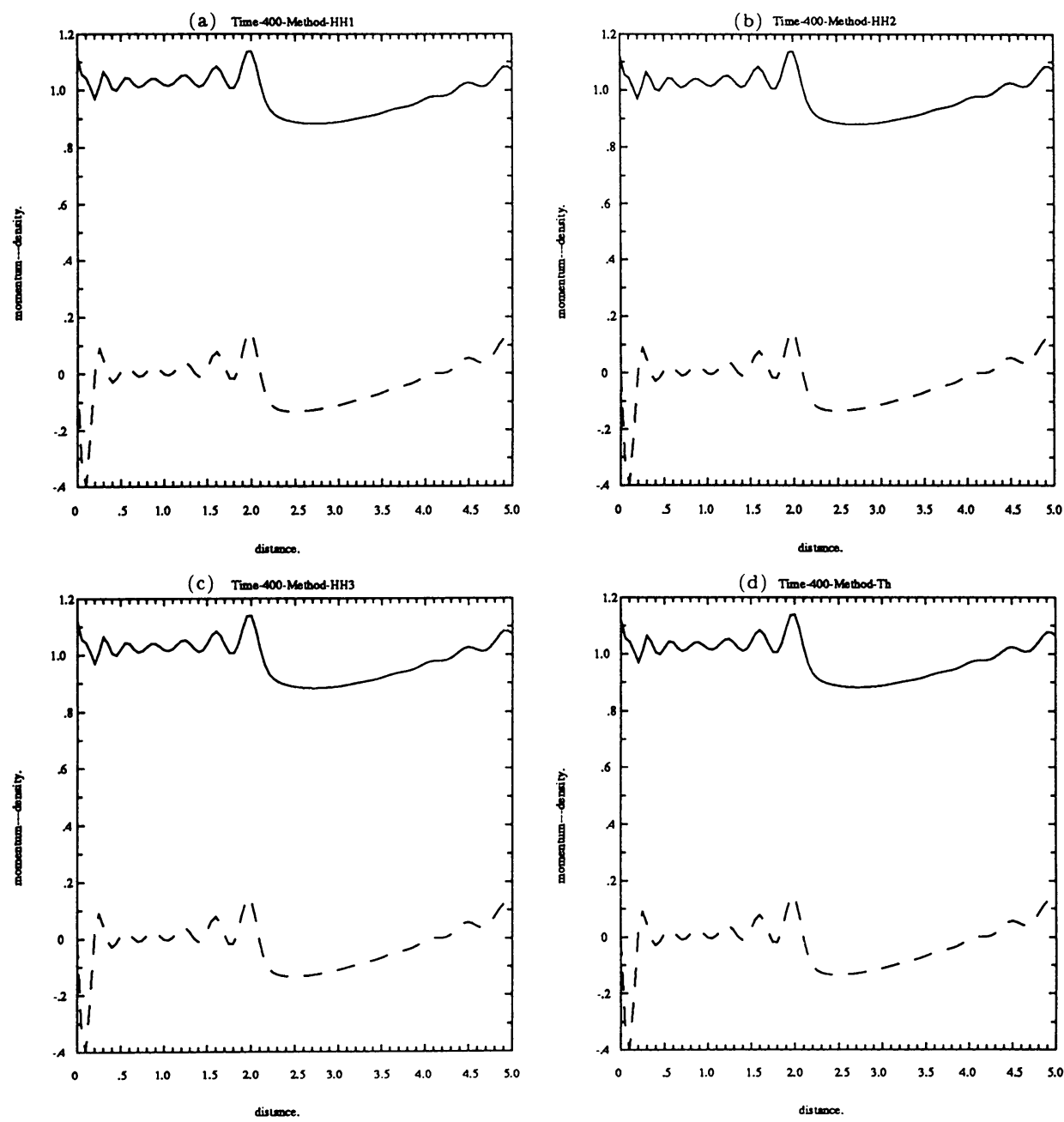

FIGURE 1

Solutions on the long interval, time $=400$.

Solid line: density

Dashed line: momentum

qualitatively the same for each boundary condition. For longer times, however, marked differences in the solutions appear. All approached a steady state. As discussed above, this is necessarily the correct steady state for HH2 and HH3. For HH1 the final density was roughly .993, an error of about .7\%. For Th it was .984, an error of $1.6 \%$.

The contrasting results are accentuated by further contraction of the computational domain. Figures 5 and 6 show the results obtained for $L=2.5$ employing boundary conditions HH3 and Th, respectively. Even at time 400, the results obtained with the nonreflecting condition are seen to be significantly in error, while those obtained with our asymptotic condition are not. Again, the steady state density found using HH3 is correct while that found using Th is off by about $12.5 \%$.

It is worthwhile to note the significant fluctuations in the variables which occur near the origin. We believe this is a natural consequence of the focussing of incoming 
(a) Time-600-Metbod-HHI

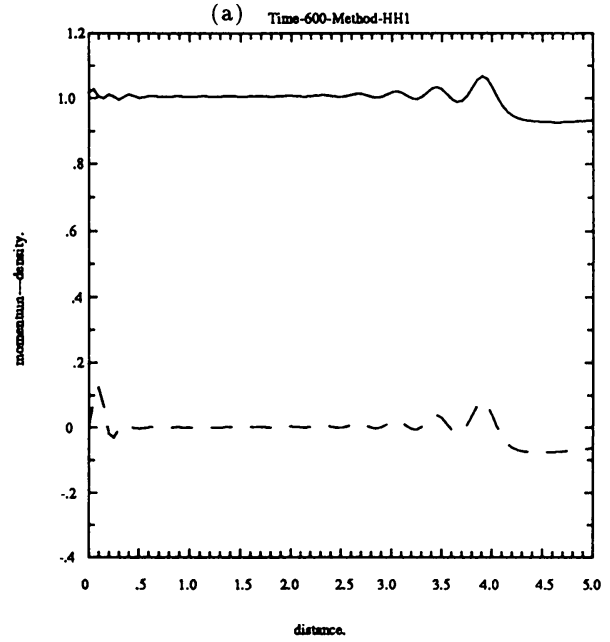

(c) Time-600-Melsod-HH3

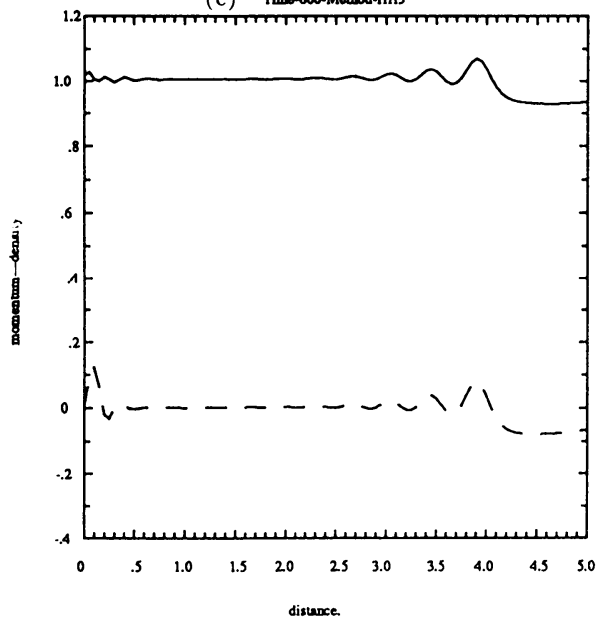

(b) Time-600-Method-HHz

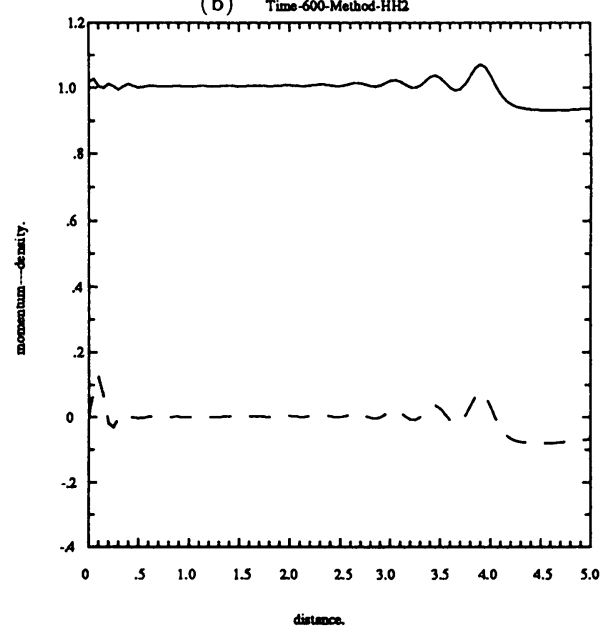

(d) Timo-600-Molood-Th

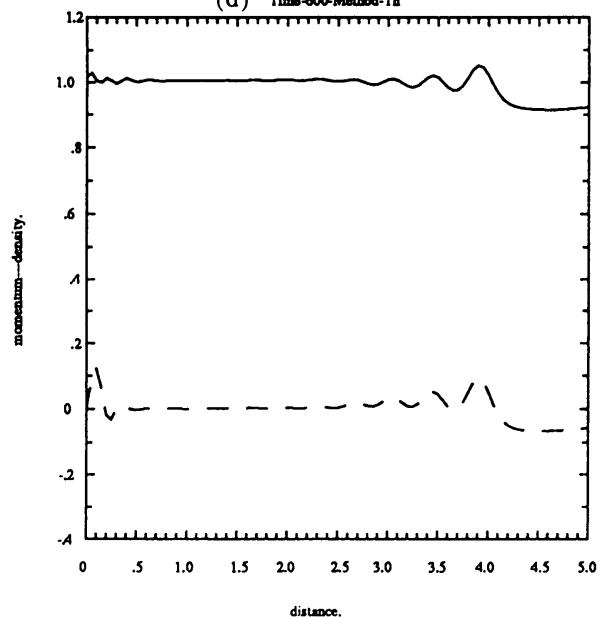

FIGURE 2

Solutions on the long interval, time $=600$.

Solid line: density

Dashed line: momentum

spherical waves. The diminished amplitude of these fluctuations resulting from $\mathrm{Th}$ on the smallest domain is evidence of its inaccurate representation of the transient solution. That is, some physical, incoming waves were not generated.

Computations were performed on a Sun Microsystem 3/260 with a floating-point accelerator. It took about 56 seconds of cpu time for the first case $(L=5)$ and 30 seconds for the second case $(L=2.5)$. In each case the total number of time steps was 2000 .

In conclusion, we have established the accuracy of our reflecting condition even when implemented on a domain of modest size. Such ideas become crucial in truly multidimensional computations. Though we have not performed any such computations, we present ideas in the next section for the generalization of our boundary conditions to nonsymmetric problems. 
(a) Time-800-Meibod-HH1

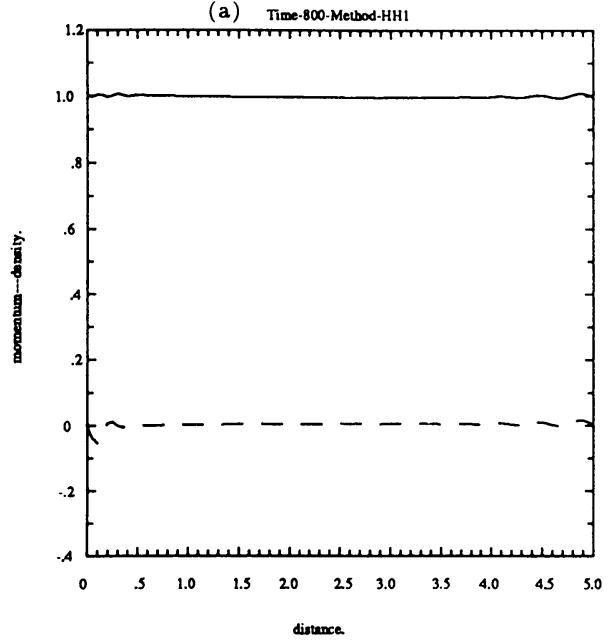

(c) Timo-800-Method-HH3

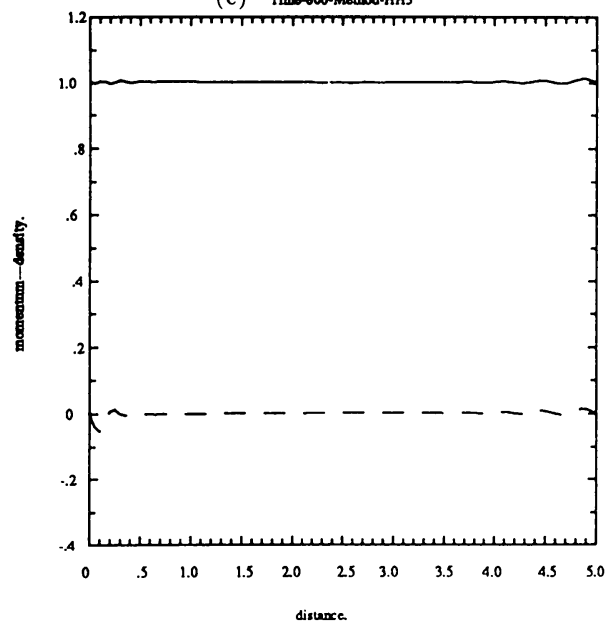

(b) Time-800-Method-HH2

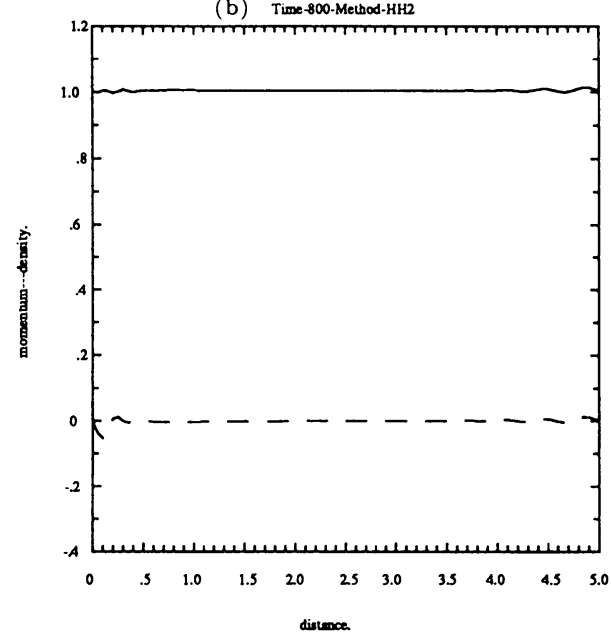

(d) Time-s00-Mechod-Th

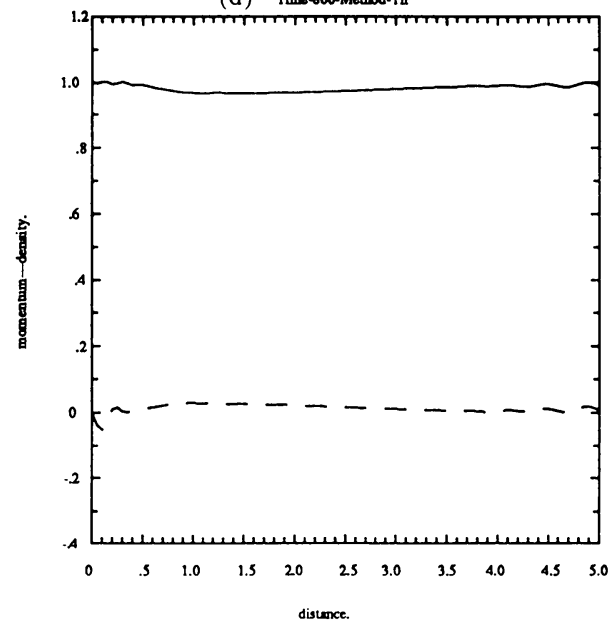

FIGURE 3

Solutions on the long interval, time $=800$.

Solid line: density

Dashed line: momentum

6. Generalizations to Nonsymmetric Flows. We now consider the Euler equations in spherical coordinates for nonsymmetric, isentropic flows. The new dependent variables are taken to be the angular momenta, $m$ and $q$ :

$$
\frac{\partial \rho}{\partial t}+\frac{\partial z}{\partial r}+\frac{1}{r} \frac{\partial m}{\partial \theta}+\frac{1}{r \sin \theta} \frac{\partial q}{\partial \phi}=g_{1}
$$

$$
\begin{array}{r}
\frac{\partial z}{\partial t}+\frac{\partial}{\partial r}\left(\frac{z^{2}}{\rho}+f(\rho)\right)+\frac{1}{r} \frac{\partial}{\partial \theta}\left(\frac{m z}{\rho}\right)+\frac{1}{r \sin \theta} \frac{\partial}{\partial \phi}\left(\frac{q z}{\rho}\right)=g_{2}, \\
\frac{\partial m}{\partial t}+\frac{\partial}{\partial r}\left(\frac{m z}{\rho}\right)+\frac{1}{r} \frac{\partial}{\partial \theta}\left(\frac{m^{2}}{\rho}+f(\rho)\right)+\frac{1}{r \sin \theta} \frac{\partial}{\partial \phi}\left(\frac{m q}{\rho}\right)=g_{3}, \\
\frac{\partial q}{\partial t}+\frac{\partial}{\partial r}\left(\frac{q z}{\rho}\right)+\frac{1}{r} \frac{\partial}{\partial \theta}\left(\frac{q m}{\rho}\right)+\frac{1}{r \sin \theta} \frac{\partial}{\partial \phi}\left(\frac{q^{2}}{\rho}+f(\rho)\right)=g_{4},
\end{array}
$$


(a) Time-1000-Method-HH1

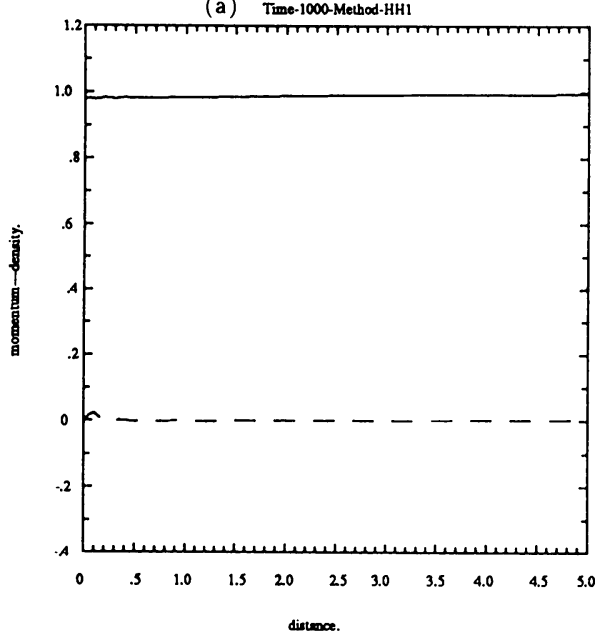

(c) Tmo-1000-Malbod-HH3

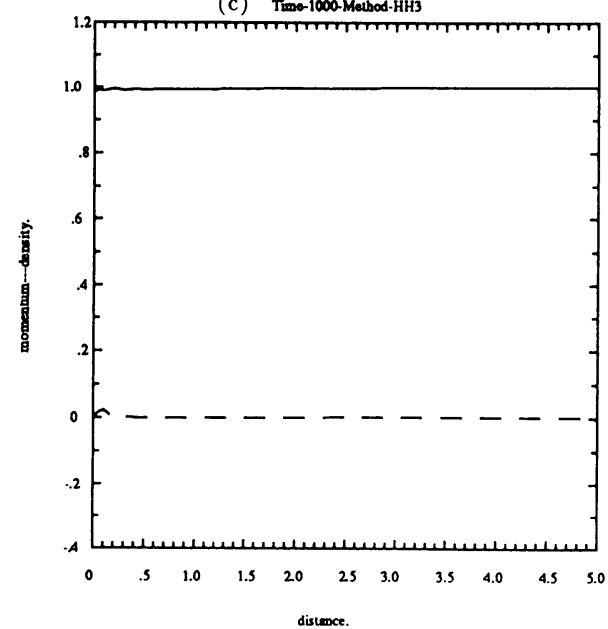

(b) Time-1000-Method-HH2

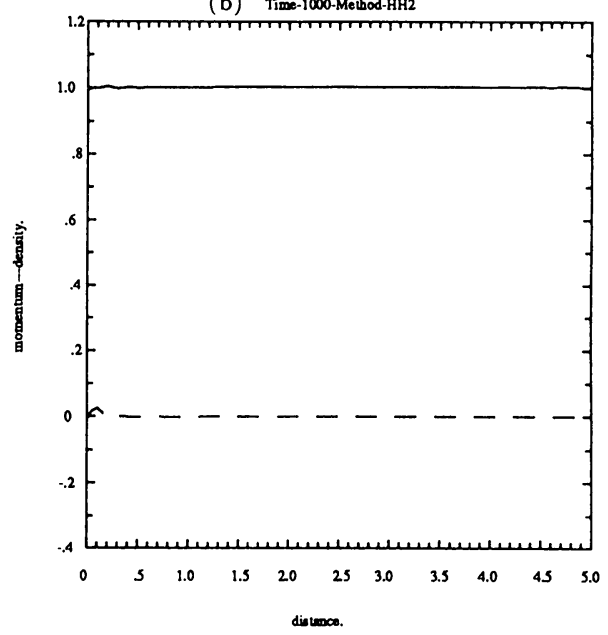

(d) The-1000-Metrod-Th

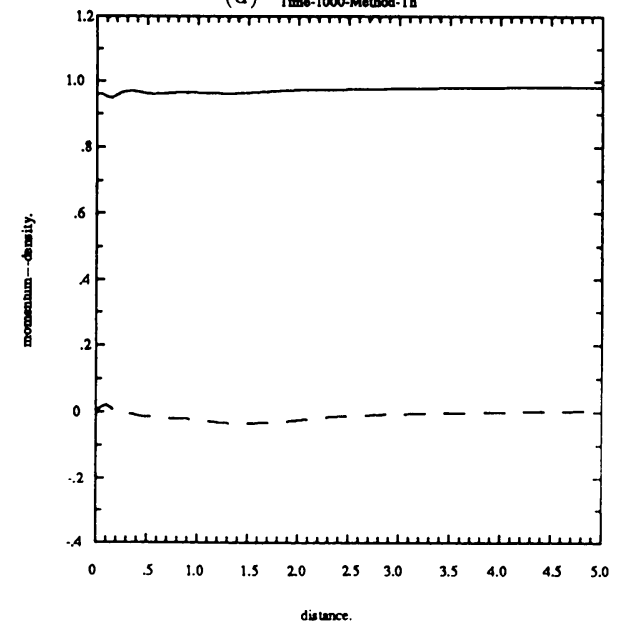

FIGURE 4

Solutions on the long interval, time $=1000$.

Solid line: density

Dashed line: momentum

where we have introduced,

$$
\begin{aligned}
& g_{1}=-\frac{2 z}{r}-\frac{m \cot \theta}{r} \\
& g_{2}=-\frac{2 z^{2}-m^{2}-q^{2}}{\rho r}-\frac{m z \cot \theta}{\rho r} \\
& g_{3}=-\frac{3 m z}{\rho r}-\frac{\cot \theta}{\rho r}\left(m^{2}-q^{2}\right) \\
& g_{4}=-\frac{3 q z}{\rho r}-\frac{2 q m \cot \theta}{\rho r}
\end{aligned}
$$

We again consider a domain exterior to a compact body and assume the initial conditions satisfy $\rho=\rho_{\infty}$ and $z=m=q=0$ for $r \geq L$. Following the construction of Section 2, we work with the symmetric Riemann variables, $R$ and $S$, as well as 

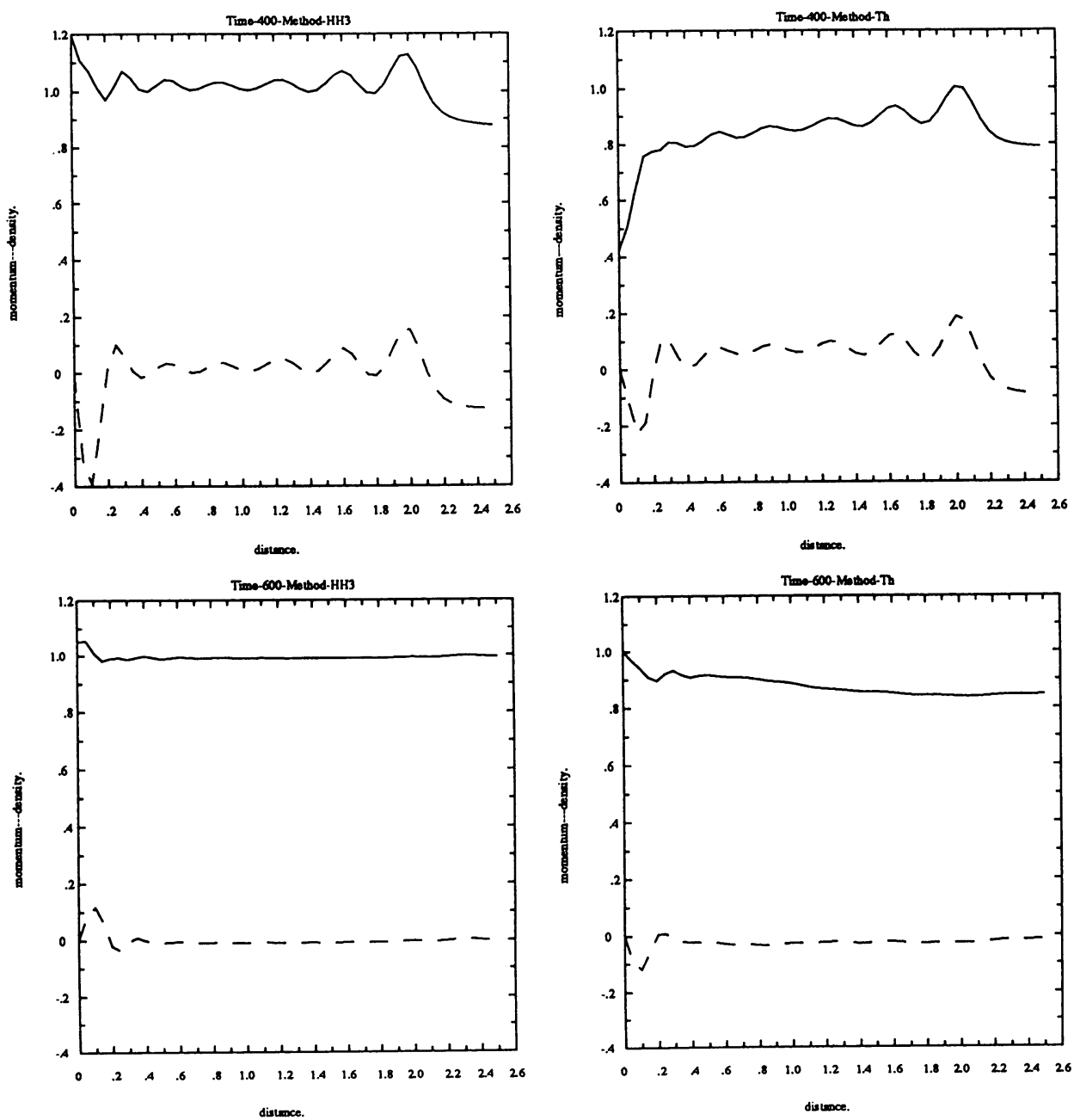

\section{FIGURE 5}

Solutions on the long interval, time $=400,600$.

Solid line: density

Dashed line: momentum

the angular momenta. The formal expansions we postulate have the form

$$
\begin{gathered}
R(r, \theta, \phi, t)=R_{0}+\frac{R_{1}(r, \theta, \phi, t)}{r}+\frac{R_{2}(r, \theta, \phi, t)}{r^{2}}+\cdots \\
S(r, \theta, \phi, t)=S_{0}+\frac{S_{1}(r, \theta, \phi, t)}{r}+\frac{S_{2}(r, \theta, \phi, t)}{r^{2}}+\cdots \\
m(r, \theta, \phi, t)=\frac{m_{2}(r, \theta, \phi, t)}{r^{2}}+\frac{m_{3}(r, \theta, \phi, t)}{r^{3}}+\cdots \\
q(r, \theta, \phi, t)=\frac{q_{2}(r, \theta, \phi, t)}{r^{2}}+\frac{q_{3}(r, \theta, \phi, t)}{r^{3}}+\cdots
\end{gathered}
$$

where, borrowing results from the linear case, we assume the angular momenta, $m$ and $q$, are $O\left(\frac{1}{r^{2}}\right)$ as $r \rightarrow \infty$. The equations for $R_{1}, R_{2}, S_{1}$ and $S_{2}$ are taken 

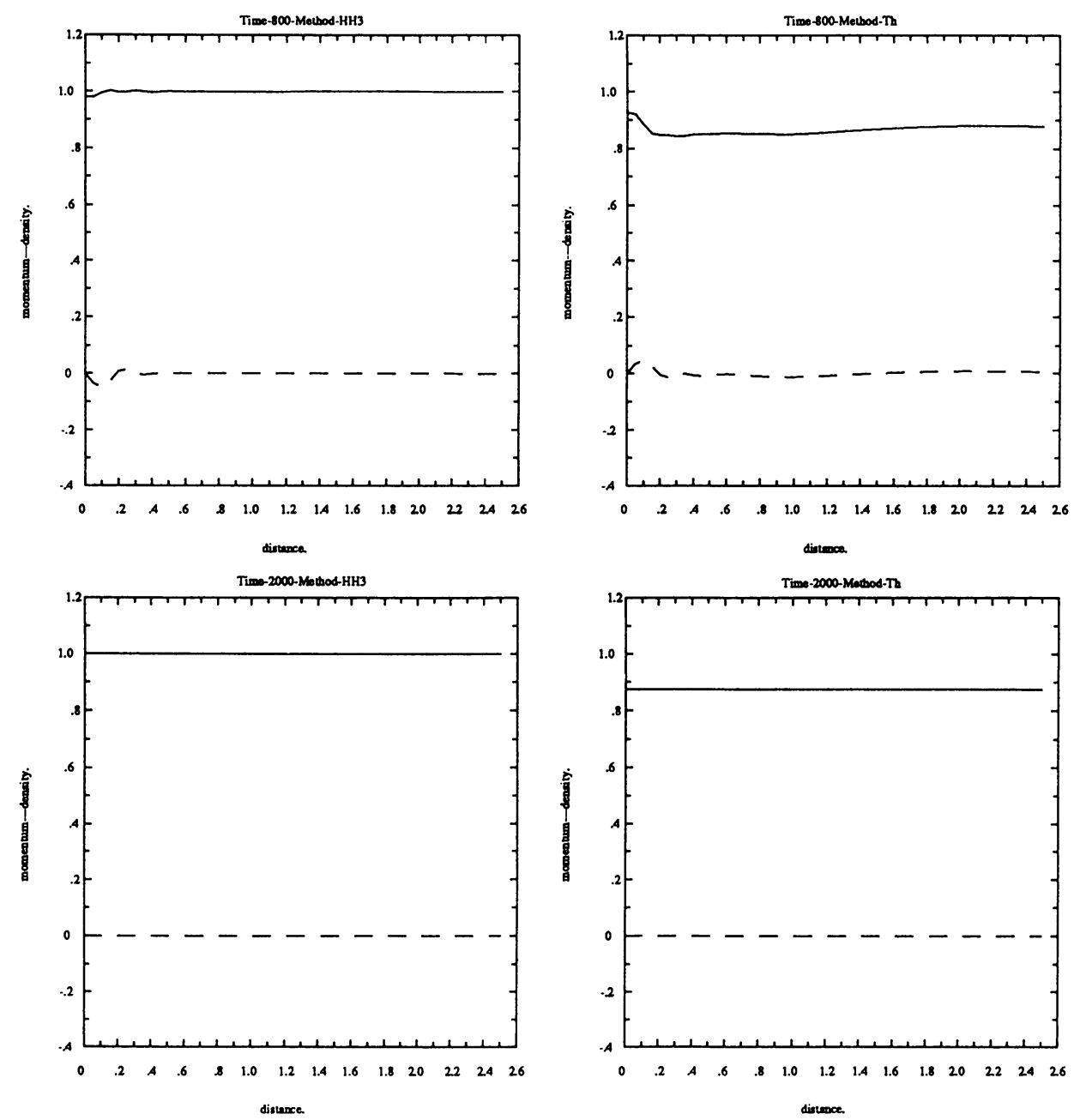

FIGURE 6

Solutions on the long interval, time $=800,2000$.

Solid line: density

Dashed line: momentum

unchanged from Section 2. This involves the neglect of lower-order terms involving $\theta$ and $\phi$ derivatives. Although not entirely consistent with our inclusion of lowerorder terms involving $\frac{\partial}{\partial r}$, this is justified by the expectation that the primary direction of propagation in the far field is the radial one. Expressions for the first corrections may then be copied from $(2.14),(2.16)$ and $(2.17)$ :

$$
\begin{gathered}
S_{1}(r, \theta, \phi, t)=0 \\
R_{1}(r, \theta, \phi, t(r, \theta, \phi, \tau))=H(\theta, \phi, \tau) \\
t(r, \theta, \phi, \tau)=\tau+\frac{r-L-B(\theta, \phi, \tau) \ln \left[\frac{r+B(\theta, \phi, \tau)}{L+B(\theta, \phi, \tau)}\right]}{\sqrt{f^{\prime}\left(\rho_{\infty}\right)}} .
\end{gathered}
$$


More importantly, the boundary conditions (2.24), (2.26) and (2.27) are unchanged. Equations for $m_{2}$ and $q_{2}$ are:

$$
\begin{gathered}
\frac{\partial m_{2}}{\partial t}+\frac{z_{1}}{r \rho_{\infty}} \frac{\partial m_{2}}{\partial r}+f^{\prime}\left(\rho_{\infty}\right) \frac{\partial \rho_{1}}{\partial \theta}=0 \\
\frac{\partial q_{2}}{\partial t}+\frac{z_{1}}{r \rho_{\infty}} \frac{\partial q_{2}}{\partial r}+\frac{f^{\prime}\left(\rho_{\infty}\right)}{\sin \theta} \frac{\partial \rho_{1}}{\partial \phi}=0
\end{gathered}
$$

Here,

and

$$
z_{1}=\frac{\rho_{\infty} R_{1}}{2}=\frac{\rho_{\infty} H}{2}
$$

$$
\rho_{1}=\frac{\rho_{\infty} R_{1}}{2 \sqrt{f^{\prime}\left(\rho_{\infty}\right)}}=\frac{\rho_{\infty} H}{2 \sqrt{f^{\prime}\left(\rho_{\infty}\right)}} .
$$

We include the $\frac{1}{r}$ term so that characteristics can be computed. In particular, at a point of outflow, $z_{1}>0$, no boundary condition for $m_{2}$ and $q_{2}$ is required. At inflow we may simply use (6.12) and (6.13) without the $r$ derivative terms to update the angular momenta. In summary we have:

$$
\frac{\partial S}{\partial t}(L, \theta, \phi, t)= \begin{cases}\frac{\sqrt{f^{\prime}\left(\rho_{\infty}\right)}\left(R(L, \theta, \phi, t)-R_{0}\right)}{2 L} & \text { or } \\ \frac{z(L, \theta, \phi, t) \sqrt{f^{\prime}\left(\rho_{\infty}\right)}}{L \rho(L, \theta, \phi, t)} & \text { or } \\ \frac{\left(G\left(\rho(L, \theta, \phi, t)-G\left(\rho_{\infty}\right)\right)\right) \sqrt{f^{\prime}\left(\rho_{\infty}\right)}}{L} & \end{cases}
$$

and, if $z(L, \theta, \phi, t) \leq 0$,

$$
\begin{gathered}
\frac{\partial m}{\partial t}(L, \theta, \phi, t)=-\frac{1}{r^{2}} \frac{\partial}{\partial \theta}[f(\rho(L, \theta, \phi, t))], \\
\frac{\partial q}{\partial t}(L, \theta, \phi, t)=-\frac{1}{r^{2} \sin \theta} \frac{\partial}{\partial \phi}[f(\rho(L, \theta, \phi, t))] .
\end{gathered}
$$

Department of Applied Mathematics and Statistics

SUNY at Stony Brook

Stony Brook, New York 11794

and

Institute for Computational Mechanics in Propulsion

NASA Lewis Research Center

Cleveland, Ohio 44135

E-mail: THAGSTROM@SBCCMAIL.BITNET

Department of Mathematical Sciences

University of Akron

Akron, Ohio 44325

E-mail: R1HARI@AKRONVM.BITNET

1. A. BAYLISS, \& E. TURKEL, "Radiation boundary condition for wave-like equations," Comm. Pure Appl. Math., v. 33, 1980, pp. 707-723.

2. B. ENGQUIST \& A. MAJDA, "Absorbing boundary conditions for the numerical simulation of waves," Math. Comp., v. 31, 1977, pp. 629-651.

3. B. GUSTAFSSON \& H.-O. KREISS, "Boundary conditions for time dependent problems with an artificial boundary," J. Comput. Phys., v. 30, 1979, pp. 333-351.

4. B. GUSTAFSSON, "Inhomogeneous conditions at open boundaries for wave propagation problems," Appl. Numer. Math., v. 4, 1988, pp. 3-19. 
5. B. GUSTAFSSON, "Far field boundary conditions for time-dependent hyperbolic systems," SIAM J. Sci. Statist. Comput., 1988. (To appear.)

6. T. HAGSTROM \& H. B. KELLER, "Exact boundary conditions at an artificial boundary for partial differential equations in cylinders," SIAM J. Math. Anal., v. 17, 1986, pp. 322-341.

7. S. I. HARIHARAN \& A. BAYLISS, "Radiation of sound from unflanged cylindrical ducts," SIAM J. Sci. Statist. Comput., v. 6, 1985, pp. 285-296.

8. G. W. HEDSTROM, "Non-reflecting boundary conditions for nonlinear hyperbolic systems," J. Comput. Phys., v. 30, 1979, pp. 222-237.

9. G. SOD, Numerical Methods in Fluid Dynamics, Cambridge Univ. Press, Cambridge, 1985.

10. K. W. ThOMPSON, "Time dependent boundary conditions for hyperbolic systems," J. Comput. Phys., v. 68, 1987, pp. 1-24.

11. G. B. Whitham, Linear and Nonlinear Waves, Wiley, New York, 1974. 